\title{
A relaxed Kaczmarz method for fuzzy linear systems
}

\author{
Ke Wang ${ }^{1}$, Shijun Zhang ${ }^{2, *}$, Shiheng Wang $^{2}$ \\ ${ }^{1}$ Department of Mathematics, Shanghai University, Shanghai 200444, P.R. China \\ ${ }^{2}$ Nanyang Vocational College of Agriculture, Nanyang 473000, P.R. China
}

\begin{abstract}
A relaxed Kaczmarz method is presented for solving a class of fuzzy linear systems of equations with crisp coefficient matrix and fuzzy right-hand side. The iterative scheme is established and the convergence theorem is provided. Numerical examples show that the method is effective.
\end{abstract}

Key words: Fuzzy linear system; relaxed Kaczmarz method; iterative scheme

\section{Introduction}

Fuzzy linear systems (FLSs) occur in many fields, such as control problems, information, physics, statistics, engineering, economics, finance and even social sciences [12]. Thus, it is significant to study the numerical methods for solving FLSs.

A general model in [12] was proposed with embedding technique by Friedman et al. for solving a class of $n \times n$ FLSs

$$
A x=y,
$$

where

$$
A=\left[\begin{array}{cccc}
a_{11} & a_{12} & \cdots & a_{1 n} \\
a_{21} & a_{22} & \cdots & a_{2 n} \\
\vdots & \vdots & \ddots & \vdots \\
a_{n 1} & a_{n 2} & \cdots & a_{n n}
\end{array}\right]
$$

is a crisp matrix, $y=\left[y_{1}, y_{2}, \cdots, y_{n}\right]^{\mathrm{T}}$ is a fuzzy vector, and $x=\left[x_{1}, x_{2}, \cdots, x_{n}\right]^{\mathrm{T}}$ is unknown. With this model, many numerical methods $[1-7,9-11,13,14,16,17,19-22]$ was developed to solve FLS (1.1).

The Kaczmarz algorithm is a popular iterative projection method [23] as it is simple to implement and suitable for parallel computing. Numbers of authors studied Kaczmarz methods for solving linear systems $[8,15,18,23]$. In this paper, a relaxed Kaczmarz algorithm is presented for fuzzy linear system (1.1).

The rest of the paper is organized as follows. Section 2 gives some basic definitions and results of FLS. In Section 3, the relaxed Kaczmarz method is established with convergence theorem. Two numerical examples in Section 4 are discussed and the conclusion is in Section 5.

\section{Preliminaries}

Generally, following [12], a fuzzy number is a pair of $(\underline{u}(r), \bar{u}(r)), 0 \leqslant r \leqslant 1$, satisfying,

\footnotetext{
${ }^{*}$ Corresponding author.
} 
- $\underline{u}(r)$ is a bounded left continuous nondecreasing function over $[0,1]$,

- $\bar{u}(r)$ is a bounded left continuous nonincreasing function over $[0,1]$,

- $\underline{u}(r) \leqslant \bar{u}(r), 0 \leqslant r \leqslant 1$.

The arithmetic operations of arbitrary fuzzy numbers $x=(\underline{x}(r), \bar{x}(r)), y=(\underline{y}(r), \bar{y}(r)), 0 \leqslant r \leqslant 1$, and real number $k$, are as follows,

(1) $x=y$ if and only if $\underline{x}(r)=\underline{y}(r)$ and $\bar{x}(r)=\bar{y}(r)$,

(2) $x+y=(\underline{x}(r)+\underline{y}(r), \bar{x}(r)+\bar{y}(r))$, and

(3) $k x= \begin{cases}(k \underline{x}(r), k \bar{x}(r)), & k \geqslant 0 \\ (k \bar{x}(r), k \underline{x}(r)), & k<0 .\end{cases}$

Definition 2.1. [12] A fuzzy number vector $X=\left(x_{1}, x_{2}, \cdots, x_{n}\right)^{\mathrm{T}}$ given by

$$
x_{i}=\left(\underline{x}_{i}(r), \bar{x}_{i}(r)\right), \quad 1 \leqslant i \leqslant n, 0 \leqslant r \leqslant 1,
$$

is called a solution of the fuzzy linear system (1.1) if

$$
\left\{\begin{array}{l}
\frac{\sum_{j=1}^{n} a_{i j} x_{j}}{\overline{\sum_{j=1}^{n} a_{i j} x_{j}}}=\sum_{j=1}^{n} \underline{a_{i j} x_{j}}=\underline{y}_{i=1}^{n} \overline{a_{i j} x_{j}}=\bar{y}_{i} .
\end{array}\right.
$$

By (2.1), Friedman et al. [12] extend FLS (1.1) to a $2 n \times 2 n$ crisp linear system

$$
S X=Y
$$

where $S=\left(s_{k l}\right), s_{k l}$ are determined as follows

$$
\begin{gathered}
a_{i j} \geqslant 0 \Rightarrow s_{i j}=a_{i j}, \quad s_{n+i, n+j}=a_{i j}, \\
a_{i j}<0 \Rightarrow s_{i, n+j}=a_{i j}, \quad s_{n+i, j}=a_{i j},
\end{gathered} \quad 1 \leqslant i, j \leqslant n,
$$

and any $s_{k l}$ which is not determined by the above items is zero, $1 \leqslant k, l \leqslant 2 n$, and

$$
X=\left[\begin{array}{c}
\underline{x}_{1} \\
\vdots \\
\underline{x}_{n} \\
\bar{x}_{1} \\
\vdots \\
\bar{x}_{n}
\end{array}\right], Y=\left[\begin{array}{c}
\underline{y}_{1} \\
\vdots \\
\underline{y}_{n} \\
\bar{y}_{1} \\
\vdots \\
\bar{y}_{n}
\end{array}\right] .
$$

Further, the matrix $S$ has the structure $\left[\begin{array}{cc}S_{1} & S_{2} \\ S_{2} & S_{1}\end{array}\right], A=S_{1}+S_{2}$, and (2.2) can be rewritten as

$$
\left\{\begin{array}{l}
S_{1} \underline{X}+S_{2} \bar{X}=\underline{Y} \\
S_{2} \underline{X}+S_{1} \bar{X}=\bar{Y}
\end{array}\right.
$$

where

$$
\underline{X}=\left[\begin{array}{c}
\underline{x}_{1} \\
\underline{x}_{2} \\
\vdots \\
\underline{x}_{n}
\end{array}\right], \bar{X}=\left[\begin{array}{c}
\bar{x}_{1} \\
\bar{x}_{2} \\
\vdots \\
\bar{x}_{n}
\end{array}\right], \underline{Y}=\left[\begin{array}{c}
\underline{y}_{1} \\
\underline{y}_{2} \\
\vdots \\
\underline{y}_{n}
\end{array}\right], \bar{Y}=\left[\begin{array}{c}
\bar{y}_{1} \\
\bar{y}_{2} \\
\vdots \\
\bar{y}_{n}
\end{array}\right]
$$

A theorem as follows indicates when FLS (1.1) has a unique solution. 
Theorem 2.2. The matrix $S$ is nonsingular if and only if the matrices $A=S_{1}+S_{2}$ and $S_{1}-S_{2}$ are both nonsingular. See [12].

In the next section, the proposed relaxed Kaczmarz method is presented for nonsingular FLS (1.1).

\section{The relaxed Kaczmarz method}

For nonsingular fuzzy linear system (2.2) or (2.3), a relaxed Kaczmarz iterative scheme can be described as follows,

$$
X_{k+1}=X_{k}+\alpha \frac{Y^{\left(i_{k}\right)}-S^{\left(i_{k}\right)} X_{k}}{\left\|S^{\left(i_{k}\right)}\right\|_{2}^{2}}\left(S^{\left(i_{k}\right)}\right)^{\mathrm{T}},
$$

and can be implemented as the following algorithm, where $0<\alpha<2$ is a relaxation parameter.

$\overline{\text { Relaxed Kaczmarz Algorithm. Given initial vectors } \underline{X}_{0}, \bar{X}_{0} \in \mathbb{R}^{n} \text {, for } k=0,1,2, \cdots \text {, the following }}$ iterative scheme is taken,

$$
\left\{\begin{array}{l}
\underline{X}_{k+1}=\underline{X}_{k}+\alpha \frac{\left(\underline{Y}-S_{2} \bar{X}_{k}\right)^{\left(i_{k}\right)}-S_{1}^{\left(i_{k}\right)} \underline{X}_{k}}{\left\|S_{1}^{\left(i_{k}\right)}\right\|_{2}^{2}}\left(S_{1}^{\left(i_{k}\right)}\right)^{\mathrm{T}}, \\
\bar{X}_{k+1}=\bar{X}_{k}+\alpha \frac{\left(\bar{Y}-S_{2} \underline{X}_{k+1}\right)^{\left(i_{k}\right)}-S_{1}^{\left(i_{k}\right)} \bar{X}_{k}}{\left\|S_{1}^{\left(i_{k}\right)}\right\|_{2}^{2}}\left(S_{1}^{\left(i_{k}\right)}\right)^{\mathrm{T}}
\end{array}\right.
$$

where $i_{k}=(k \bmod n)+1,(\cdot)^{\left(i_{k}\right)}$ denotes the $i_{k}$ th row of a matrix.

The convergence result for method (3.2) is as the following theorem.

Theorem 3.1. Suppose that fuzzy linear system (2.2) or (2.3) is consistent. Then the iterative sequence $\left.\left.\left\{\left[\underline{\underline{X}}_{k}\right]\right]_{k}\right]\right\}_{k=0}^{\infty}$, generated by the relaxed Kaczmarz method (3.2) starting from an initial guess $\left[{\underline{X_{0}}}_{0}\right]$ with $\underline{X}_{0}$ and $\bar{X}_{0}$ in the column space of $S_{2}$, converges to the unique solution $\left[{\underline{X}}_{*}\right]=\left[\begin{array}{cc}S_{1} & S_{2} \\ S_{2} & S_{1}\end{array}\right]^{-1}\left[\frac{Y}{\bar{Y}}\right]$ of $(2.2)$. Moreover, the solution error for the iteration sequence is

$$
\left\|X_{k+1}-X_{*}\right\|_{2}^{2} \leqslant\left(1-\alpha(2-\alpha) \frac{\lambda_{\min }\left(S^{\mathrm{T}} S\right)}{\|S\|_{F}^{2}}\right)\left\|X_{k}-X_{*}\right\|_{2}^{2},
$$

where $\lambda_{\min }(\cdot)$ is the smallest nonzero eigenvalues of a matrix, $X_{k}=\left[\underline{\underline{X}}_{k}\right]$, and $X_{*}=\left[{\underline{X}}_{*}\right]$.

$$
\begin{aligned}
& \text { Proof. Take } P^{\left(i_{k}\right)}=\left(\frac{S^{\left(i_{k}\right)}}{\left\|S^{\left(i_{k}\right)}\right\|_{2}}\right)^{\mathrm{T}}\left(\frac{S^{\left(i_{k}\right)}}{\left\|S^{\left(i_{k}\right)}\right\|_{2}}\right) \text {, thus } \\
& \qquad \begin{aligned}
X_{k+1}-X_{*} & =X_{k}-X_{*}+\alpha \frac{Y^{\left(i_{k}\right)}-S^{\left(i_{k}\right)} X_{k}}{\left\|S^{\left(i_{k}\right)}\right\|_{2}^{2}}\left(S^{\left(i_{k}\right)}\right)^{\mathrm{T}} \\
& =X_{k}-X_{*}-\alpha\left(S^{\left(i_{k}\right)}\right)^{\mathrm{T}} \frac{S^{\left(i_{k}\right)}\left(X_{k}-X_{*}\right)}{\left\|S^{\left(i_{k}\right)}\right\|_{2}^{2}} \\
& =\left(I-\alpha P^{\left(i_{k}\right)}\right)\left(X_{k}-X_{*}\right),
\end{aligned}
\end{aligned}
$$


then

$$
\begin{aligned}
\left\|X_{k+1}-X_{*}\right\|_{2}^{2} & =\left(X_{k}-X_{*}\right)^{\mathrm{T}}\left(I-\alpha P^{\left(i_{k}\right)}\right)^{2}\left(X_{k}-X_{*}\right) \\
& =\left(X_{k}-X_{*}\right)^{\mathrm{T}}\left(I-\alpha(2-\alpha) P^{\left(i_{k}\right)}\right)\left(X_{k}-X_{*}\right) \\
& =\left\|X_{k}-X_{*}\right\|_{2}^{2}-\alpha(2-\alpha)\left(X_{k}-X_{*}\right)^{\mathrm{T}} P^{\left(i_{k}\right)}\left(X_{k}-X_{*}\right),
\end{aligned}
$$

and

$$
\begin{aligned}
\left(X_{k}-X_{*}\right)^{\mathrm{T}} P^{\left(i_{k}\right)}\left(X_{k}-X_{*}\right) & =\frac{\left(X_{k}-X_{*}\right)^{\mathrm{T}}\left(S^{\left(i_{k}\right)}\right)^{\mathrm{T}} S^{\left(i_{k}\right)}\left(X_{k}-X_{*}\right)}{\left\|S^{\left(i_{k}\right)}\right\|_{2}^{2}} \\
& =\frac{\left|S^{\left(i_{k}\right)}\left(X_{k}-X_{*}\right)\right|^{2}}{\left\|S^{\left(i_{k}\right)}\right\|_{2}^{2}} \geqslant \frac{\left\|S\left(X_{k}-X_{*}\right)\right\|_{2}^{2}}{\|S\|_{F}^{2}} .
\end{aligned}
$$

As $x_{0}$ is in the column space of $S$, from [23], it holds that $\left\|S\left(X_{k}-X_{*}\right)\right\|_{2}^{2} \geqslant \lambda_{\min }\left(S^{\mathrm{T}} S\right)\left\|\left(X_{k}-X_{*}\right)\right\|_{2}^{2}$. Therefore, the following is obtained

$$
\left\|X_{k+1}-X_{*}\right\|_{2}^{2} \leqslant\left(1-\alpha(2-\alpha) \frac{\lambda_{\min }\left(S^{\mathrm{T}} S\right)}{\|S\|_{F}^{2}}\right)\left\|X_{k}-X_{*}\right\|_{2}^{2} .
$$

The proof is completed.

\section{Numerical Examples}

This section gives two examples to show the effectiveness of the relaxed Kaczmarz method. All implements using Matlab 7 run in a Windows 7 DELL laptop with Intel 2.80GHz CPU and 8.00GB RAM. In the numerical experiments, the initial guess is 0 and the stopping criterion is

$$
\left\|R_{k}\right\|_{2}<10^{-6}
$$

where $R_{k}$ is the residual vector after $k$ iterations, i.e., $R_{k}=Y-S X_{k}$.

In the tables, $x_{a}$ and $x_{b}$ mean that $S X=Y$ is solved as two numeric systems

$$
S\left[\begin{array}{c}
x_{a 1} \\
x_{a 2} \\
\vdots \\
x_{a, 2 n}
\end{array}\right]=\left[\begin{array}{c}
y_{a 1} \\
y_{a 2} \\
\vdots \\
y_{a, 2 n}
\end{array}\right] \text { and } S\left[\begin{array}{c}
x_{b 1} \\
x_{b 2} \\
\vdots \\
x_{b, 2 n}
\end{array}\right]=\left[\begin{array}{c}
y_{b 1} \\
y_{b 2} \\
\vdots \\
y_{b, 2 n}
\end{array}\right]
$$

not one symbolic system

$$
S\left[\begin{array}{c}
x_{a 1}+x_{b 1} r \\
x_{a 2}+x_{b 2} r \\
\vdots \\
x_{a, 2 n}+x_{b, 2 n} r
\end{array}\right]=\left[\begin{array}{c}
y_{a 1}+y_{b 1} r \\
y_{a 2}+y_{b 2} r \\
\vdots \\
y_{a, 2 n}+y_{b, 2 n} r
\end{array}\right]
$$

in the actual calculations. 
Example 4.1. Consider $n \times n$ fuzzy linear system $A x=y$ with

$$
A=\left[\begin{array}{ccccccccc}
8 & -1 & -1 & -1 & & & & & \\
-1 & 8 & -1 & -1 & \ddots & & & & \\
-1 & -1 & 8 & -1 & \ddots & \ddots & & & \\
-1 & -1 & -1 & 8 & \ddots & \ddots & \ddots & & \\
& \ddots & \ddots & \ddots & \ddots & \ddots & \ddots & \ddots & \\
& & \ddots & \ddots & \ddots & 8 & -1 & -1 & -1 \\
& & & \ddots & \ddots & -1 & 8 & -1 & -1 \\
& & & & \ddots & -1 & -1 & 8 & -1 \\
& & & & & -1 & -1 & -1 & 8
\end{array}\right]
$$

and

$$
y=\left[\begin{array}{c}
(2+r, 2+r) \\
(2+r, 2+r) \\
\vdots \\
(2+r, 2+r)
\end{array}\right]
$$

Table 1

Iterations (IT) and Residual (RES) for Example 4.1, $\alpha=0.8$

\begin{tabular}{ccccc}
\hline$n$ & $\mathrm{IT}_{x_{a}}$ & $\mathrm{RES}_{x_{a}}$ & $\mathrm{IT}_{x_{b}}$ & $\mathrm{RES}_{x_{b}}$ \\
\hline 16 & 617 & $9.6319 \mathrm{e}-007$ & 590 & $9.9255 \mathrm{e}-007$ \\
32 & 1456 & $9.8052 \mathrm{e}-007$ & 1394 & $9.8950 \mathrm{e}-007$ \\
64 & 3149 & $9.9849 \mathrm{e}-007$ & 3021 & $9.9572 \mathrm{e}-007$ \\
128 & 6494 & $9.9873 \mathrm{e}-007$ & 6235 & $9.9828 \mathrm{e}-007$ \\
256 & 13367 & $9.9960 \mathrm{e}-007$ & 12840 & $9.9995 \mathrm{e}-007$ \\
512 & 27327 & $9.9969 \mathrm{e}-007$ & 26272 & $9.9993 \mathrm{e}-007$ \\
\hline
\end{tabular}

Table 2

Iterations (IT) and Residual (RES) for Example 4.1, $\alpha=1$

\begin{tabular}{ccccc}
\hline$n$ & $\mathrm{IT}_{x_{a}}$ & RES $_{x_{a}}$ & $\mathrm{IT}_{x_{b}}$ & $\mathrm{RES}_{x_{b}}$ \\
\hline 16 & 387 & $9.8857 \mathrm{e}-007$ & 371 & $9.9516 \mathrm{e}-007$ \\
32 & 937 & $9.8926 \mathrm{e}-007$ & 903 & $9.7518 \mathrm{e}-007$ \\
64 & 2063 & $9.9769 \mathrm{e}-007$ & 1959 & $9.9717 \mathrm{e}-007$ \\
128 & 4283 & $9.9940 \mathrm{e}-007$ & 4119 & $9.9840 \mathrm{e}-007$ \\
256 & 8810 & $9.9827 \mathrm{e}-007$ & 8420 & $9.9985 \mathrm{e}-007$ \\
512 & 18001 & $9.9982 \mathrm{e}-007$ & 17298 & $9.9991 \mathrm{e}-007$ \\
\hline
\end{tabular}

Table 3

Iterations (IT) and Residual (RES) for Example 4.1, $\alpha=1.2$

\begin{tabular}{ccccc}
\hline$n$ & $\mathrm{IT}_{x_{a}}$ & RES $_{x_{a}}$ & $\mathrm{IT}_{x_{b}}$ & $\mathrm{RES}_{x_{b}}$ \\
\hline 16 & 193 & $9.1000 \mathrm{e}-007$ & 183 & $9.0818 \mathrm{e}-007$ \\
32 & 524 & $9.7139 \mathrm{e}-007$ & 518 & $8.8871 \mathrm{e}-007$ \\
64 & 1301 & $9.8178 \mathrm{e}-007$ & 1242 & $9.7986 \mathrm{e}-007$ \\
128 & 2758 & $9.9848 \mathrm{e}-007$ & 2639 & $9.9766 \mathrm{e}-007$ \\
256 & 5715 & $9.9972 \mathrm{e}-007$ & 5494 & $9.9795 \mathrm{e}-007$ \\
512 & 11670 & $9.9882 \mathrm{e}-007$ & 11193 & $9.9685 \mathrm{e}-007$ \\
\hline
\end{tabular}


Example 4.2. Consider $n^{2} \times n^{2}$ fuzzy linear system $A x=y$ with

$$
A=\left[\begin{array}{ccccc}
D & B^{\mathrm{T}} & & & \\
B & D & \ddots & & \\
& \ddots & \ddots & \ddots & \\
& & \ddots & D & B^{\mathrm{T}} \\
& & & B & D
\end{array}\right]
$$

where

$$
B=\left[\begin{array}{ccccc}
0.5 & -0.25 & & & \\
-0.25 & 0.5 & \ddots & & \\
& \ddots & \ddots & \ddots & \\
& & \ddots & 0.5 & -0.25 \\
& & & -0.25 & 0.5
\end{array}\right], D=\left[\begin{array}{ccccc}
5 & -1 & & & \\
-1 & 5 & \ddots & \\
& \ddots & \ddots & \ddots & \\
& & \ddots & 5 & -1 \\
& & & -1 & 5
\end{array}\right]
$$

and

$$
y=\left[\begin{array}{c}
(1+r, 1+r) \\
(2+r, 2+r) \\
\vdots \\
\left(n^{2}+r, n^{2}+r\right)
\end{array}\right] .
$$

Table 4

Iterations (IT) and Residual (RES) for Example 4.2, $\alpha=0.98$

\begin{tabular}{ccccc}
\hline$n$ & $\mathrm{IT}_{x_{a}}$ & $\mathrm{RES}_{x_{a}}$ & $\mathrm{IT}_{x_{b}}$ & $\mathrm{RES}_{x_{b}}$ \\
\hline 10 & 1772 & $8.9357 \mathrm{e}-007$ & 1363 & $9.9337 \mathrm{e}-007$ \\
15 & 4248 & $9.9182 \mathrm{e}-007$ & 3121 & $9.8859 \mathrm{e}-007$ \\
20 & 7948 & $9.8889 \mathrm{e}-007$ & 5570 & $9.9014 \mathrm{e}-007$ \\
25 & 13014 & $9.9777 \mathrm{e}-007$ & 8720 & $9.9602 \mathrm{e}-007$ \\
30 & 18828 & $9.9025 \mathrm{e}-007$ & 12587 & $9.9366 \mathrm{e}-007$ \\
35 & 26732 & $9.9937 \mathrm{e}-007$ & 17492 & $9.9987 \mathrm{e}-007$ \\
\hline
\end{tabular}

Table 5

Iterations (IT) and Residual (RES) for Example 4.2, $\alpha=1$

\begin{tabular}{ccccc}
\hline$n$ & $\mathrm{IT}_{x_{a}}$ & $\mathrm{RES}_{x_{a}}$ & $\mathrm{IT}_{x_{b}}$ & $\mathrm{RES}_{x_{b}}$ \\
\hline 10 & 1734 & $9.6080 \mathrm{e}-007$ & 1283 & $9.3918 \mathrm{e}-007$ \\
15 & 4193 & $9.6755 \mathrm{e}-007$ & 3023 & $9.9710 \mathrm{e}-007$ \\
20 & 7711 & $9.9794 \mathrm{e}-007$ & 5543 & $9.9329 \mathrm{e}-007$ \\
25 & 12434 & $9.9822 \mathrm{e}-007$ & 8704 & $9.9622 \mathrm{e}-007$ \\
30 & 18408 & $9.9942 \mathrm{e}-007$ & 12563 & $9.9846 \mathrm{e}-007$ \\
35 & 25599 & $9.9671 \mathrm{e}-007$ & 17283 & $9.9737 \mathrm{e}-007$ \\
\hline
\end{tabular}

Table 6

Iterations (IT) and Residual (RES) for Example 4.2, $\alpha=1.01$ 


\begin{tabular}{lcccc}
\hline$n$ & $\mathrm{IT}_{x_{a}}$ & RES $_{x_{a}}$ & $\mathrm{IT}_{x_{b}}$ & RES $_{x_{b}}$ \\
\hline 10 & 1713 & $9.8178 \mathrm{e}-007$ & 1275 & $9.8845 \mathrm{e}-007$ \\
15 & 4135 & $9.9971 \mathrm{e}-007$ & 2993 & $9.9549 \mathrm{e}-007$ \\
20 & 7707 & $9.9933 \mathrm{e}-007$ & 5544 & $9.9592 \mathrm{e}-007$ \\
25 & 12546 & $9.9998 \mathrm{e}-007$ & 8793 & $9.9290 \mathrm{e}-007$ \\
30 & 18441 & $9.9335 \mathrm{e}-007$ & 12833 & $9.9414 \mathrm{e}-007$ \\
35 & 25672 & $9.9948 \mathrm{e}-007$ & 17633 & $9.9540 \mathrm{e}-007$ \\
\hline
\end{tabular}

Tables 1-6 give the number of iterations (IT) and the residual of the stopping step (RES). As $n$ increases, the method requires more iterations, and with different $\alpha$ the method has different convergence rate, thus, improvement should be made and the optimal parameter should be studied to change the convergence.

\section{Conclusion}

A relaxed Kaczmarz method is presented for solving $n \times n$ fuzzy linear system. The numerical results show that the method is effective. Further work would be improving the method and comparing with other methods, also exploring the optimal relaxation parameter.

\section{Compliance with Ethical Standards}

Ethical approval This article does not contain any studies with human participants or animals performed by any of the authors.

Funding Supported by Key Scientific Research Project of Colleges and Universities in Henan Province (20B110012), China.

Conflict of interest The authors declare that there is no conflict of interest regarding the publication of this article.

Informed consent From all individual participants included in the study.

\section{Authorship contributions}

All authors contributed to the study conception and design.

\section{References}

[1] S. Abbasbandy, R. Ezzati, and A. Jafarian, " $L U$ decomposition method for solving fuzzy system of linear equations," Appl. Math. Comput., 172, 633-643 (2006).

[2] S. Abbasbandy and A. Jafarian, "Steepest descent method for system of fuzzy linear equations," Appl. Math. Comput., 175, 823-833 (2006).

[3] S. Abbasbandy, A. Jafarian, and R. Ezzati, "Conjugate gradient method for fuzzy symmetric positive definite system of linear equations," Appl. Math. Comput., 171, 1184-1191 (2005).

[4] M. Akram, T. Allahviranloo, W. Pedrycz and M. Ali, "Methods for solving LR-bipolar fuzzy linear systems," Soft Comput., 25, 85-108 (2021). 
[5] T. Allahviranloo, "Numerical methods for fuzzy system of linear equations," Appl. Math. Comput., 155, 493-502 (2004).

[6] T. Allahviranloo, "Successive over relaxation iterative method for fuzzy system of linear equations," Appl. Math. Comput., 162, 189-196 (2005).

[7] T. Allahviranloo, "The Adomian decomposition method for fuzzy system of linear equations," Appl. Math. Comput., 163, 553-563 (2005).

[8] Z.-Z. Bai, W.-T. Wu, "On relaxed greedy randomized Kaczmarz methods for solving large sparse linear systems," Applied Mathematics Letters, 83, 21-26 (2018).

[9] M. Dehghan and B. Hashemi, "Iterative solution of fuzzy linear systems," Appl. Math. Comput., 175, 645-674 (2006).

[10] R. Ezzati, "Solving fuzzy linear systems," Soft Comput., 15, 193-197 (2011).

[11] M.A. Fariborzi Araghi and A. Fallahzadeh, "Inherited LU factorization for solving fuzzy system of linear equations," Soft Comput., 17, 159-163 (2013).

[12] M. Friedman, M. Ming, and A. Kandel, "Fuzzy linear systems," Fuzzy Sets and Systems, 96, 201-209 (1998).

[13] A.N.A. Koam, M. Akram, G. Muhammad and N. Hussain, "LU Decomposition Scheme for Solving m-Polar Fuzzy System of Linear Equations," Math. Probl. Eng., 2020, 8384593, (2020).

[14] J. Li, W. Li, and X. Kong, "A new algorithm model for solving fuzzy linear systems," Southeast Asian Bull. Math., 34, 121-132 (2010).

[15] Y. Liu, C.-Q. Gu, "Variant of greedy randomized Kaczmarz for ridge regression," Applied Numerical Mathematics, 143 223-246 (2019).

[16] S.-X. Miao, B. Zheng, and K. Wang, "Block SOR methods for fuzzy linear systems," J. Appl. Math. Comput., 26, 201-218 (2008).

[17] S.H. Nasseri, M. Matinfar, and M. Sohrabi, "QR-decomposition method for solving fuzzy system of linear equations," Int. J. Math. Comput., 4, 129-136 (2009).

[18] Y.-Q. Niu, B. Zheng, "A greedy block Kaczmarz algorithm for solving large-scale linear systems," Applied Mathematics Letters, 104, 106294 (2020).

[19] K. Wang and Y. Wu, "Uzawa-SOR method for fuzzy linear system," International Journal of Information and Computer Science, 1, 36-39 (2012).

[20] K. Wang and B. Zheng, "Symmetric successive overrelaxation methods for fuzzy linear systems," Appl. Math. Comput., 175, 891-901 (2006).

[21] K. Wang and B. Zheng, "Block iterative methods for fuzzy linear systems," J. Appl. Math. Comput., 25, 119-136 (2007).

[22] J.-F. Yin and K. Wang, "Splitting iterative methods for fuzzy system of linear equations," Comput. Math. Model., 20, 326-335 (2009).

[23] J.-J. Zhang, "A new greedy Kaczmarz algorithm for the solution of very large linear systems," - it Applied Mathematics Letters 91, 207-212 (2019). 